\title{
On the Chern-Gauss-Bonnet Theorem and Conformally Twisted Spectral Triples for $C^{*}$-Dynamical Systems
}

\author{
Farzad FATHIZADEH ${ }^{\dagger}$ and Olivier GABRIEL ${ }^{\ddagger}$ \\ $\dagger$ Department of Mathematics, Mail Code 253-37, California Institute of Technology, \\ 1200 E. California Blvd., Pasadena, CA 91125, USA \\ E-mail: farzadf@caltech.edu \\ ¥ University of Copenhagen, Universitetsparken 5, 2100 København Ø, Denmark \\ E-mail: olivier.gabriel.geom@gmail.com \\ URL: http://oliviergabriel.eu/
}

Received October 26, 2015, in final form February 04, 2016; Published online February 10, 2016 http://dx.doi.org/10.3842/SIGMA.2016.016

\begin{abstract}
The analog of the Chern-Gauss-Bonnet theorem is studied for a $C^{*}$-dynamical system consisting of a $C^{*}$-algebra $A$ equipped with an ergodic action of a compact Lie group $G$. The structure of the Lie algebra $\mathfrak{g}$ of $G$ is used to interpret the Chevalley-Eilenberg complex with coefficients in the smooth subalgebra $\mathcal{A} \subset A$ as noncommutative differential forms on the dynamical system. We conformally perturb the standard metric, which is associated with the unique $G$-invariant state on $A$, by means of a Weyl conformal factor given by a positive invertible element of the algebra, and consider the Hermitian structure that it induces on the complex. A Hodge decomposition theorem is proved, which allows us to relate the Euler characteristic of the complex to the index properties of a Hodge-de Rham operator for the perturbed metric. This operator, which is shown to be selfadjoint, is a key ingredient in our construction of a spectral triple on $\mathcal{A}$ and a twisted spectral triple on its opposite algebra. The conformal invariance of the Euler characteristic is interpreted as an indication of the Chern-Gauss-Bonnet theorem in this setting. The spectral triples encoding the conformally perturbed metrics are shown to enjoy the same spectral summability properties as the unperturbed case.
\end{abstract}

Key words: $C^{*}$-dynamical systems; ergodic action; invariant state; conformal factor; Hodgede Rham operator; noncommutative de Rham complex; Euler characteristic; Chern-GaussBonnet theorem; ordinary and twisted spectral triples; unbounded selfadjoint operators; spectral dimension

2010 Mathematics Subject Classification: 58B34; 47B25; 46L05

\section{Introduction}

In noncommutative geometry [13, 14], $C^{*}$-dynamical systems $(A, G, \alpha)$ have been long studied from a differentiable point of view starting with extending the basic notions of differential geometry and differential topology to a differential structure on a $C^{*}$-algebra $A$ endowed with an action $\alpha: G \rightarrow \operatorname{Aut}(A)$ of a Lie group $G$. That is, the notion of a connection, a vector bundle, and Chern classes were introduced for such a dynamical system, a pseudodifferential calculus was developed and the analog of the Atiyah-Singer index theorem was proved in [12]. The noncommutative two torus $\mathbb{T}_{\theta}^{2}$ has been one of the main motivating examples for these developments. In [55], this line of investigation has been taken further focusing on general compact Lie groups and index theory.

Following the seminal work of Connes and Tretkoff on the Gauss-Bonnet theorem for $\mathbb{T}_{\theta}^{2}[18]$ and its extension in [25] concerning general translation invariant conformal structures, local 
differential geometry of non-flat noncommutative tori has been a subject of increasing interest in recent years [3, 17, 26, 27, 28, 45]. A Weyl conformal factor may be used to perturb a flat metric on noncommutative tori, and Connes' pseudodifferential calculus [12] can be employed along with noncommutative computational methods to carry out calculation of scalar curvature and to investigate the related differential geometric statements, see also [20] for an asymmetric perturbation of the metric.

The idea and the techniques were indeed initiated in a preprint [11], where with the help of complicated modified logarithmic functions and a modular automorphism, an expression for the value $\zeta(0)$ of the spectral zeta function of the Laplacian of a curved metric on $\mathbb{T}_{\theta}^{2}$ was written. The vanishing of this expression is interpreted as the Gauss-Bonnet theorem [18], which was suggested by the developments in the following intimately related theories. In fact, the spectral action principle [9], in particular the related calculations in the presence of a dilaton [10], and the theory of twisted spectral triples, which arise naturally in noncommutative conformal geometry $[16,49]$, indicate independence of $\zeta(0)$ from the conformal factor.

Connes' index formula for Fredholm modules, which involves cyclic cohomology, is quite broad [13]. It asserts that given a finitely summable Fredholm module over an algebra, the analytic index, given by pairing a $K$-homology and a $K$-theory element of the algebra, coincides with the topological index, which pairs the corresponding elements in periodic cyclic cohomology and homology obtained by the Chern-Connes characters. The local index formula of Connes and Moscovici [15] gives a local formula based on residue trace functionals, which is in the same cyclic cohomology class as the Chern-Connes character, and has the advantage that one can perform explicit computations with it (see also [34]). The residue trace functionals are intimately related to the spectral formulation of Wodzicki's noncommutative residue [59, 60]. In fact, the formulation of the noncommutative residue as an integration over the cosphere bundle of a manifold also is important for explicit computations with noncommutative geometric spaces, see $[22,28,29]$ for a related treatment on noncommutative tori.

The notion of a twisted spectral triple introduced by Connes and Moscovici [16] allows to incorporate a variety of new examples, in particular type III examples in the sense of the Murrayvon Neumann classification of operator algebras. They have shown that the Chern-Connes character of a finitely summable twisted spectral triple is an ordinary cyclic cocycle and enjoys an index pairing with $K$-theory. Also, they have constructed a local Hochschild cocycle, which indicates that the ground is prepared for extending the local index formula to the twisted case. This was carried out in [47] for a particular class of twisted spectral triples; the analog of Connes' character formula was investigated in [24] for the examples. For treatments using twisted cyclic theory, in particular for relations of the theory with Cuntz algebra [19] and quantum groups, we refer to $[6,7,8]$, see also [37, 38]. More recent works related to the twisted version of spectral triples reveal their connections with the Bost-Connes system, Riemann surfaces and graphs [33], and with the standard model of particle physics [21]. Twisted spectral triples associated with crossed product algebras are studied in [16, 36, 47], see also [23] for an algebraic treatment.

Ergodic actions of compact groups on operator algebras are well-studied in the von Neumann setting (see, e.g., $[56,57,58]$ ) and in a $C^{*}$-algebraic context. They were first introduced for $C^{*}$-algebras by E. Størmer [54] and this initial effort was expanded in various articles. Let us just mention two of them:

- In their article [1], Albeverio and Høegh-Krohn investigate in particular ergodic actions on commutative $C^{*}$-algebras $A=C(X)$ and prove that they correspond to continuous transitive actions on $X$.

- The article [35] by Høegh-Krohn, Landstad and Størmer proves that if $G$ acts ergodically on a unital $C^{*}$-algebra $A$, its unique $G$-invariant state is actually a trace. 
The article [52] was the first to suggest in 1998 that ergodic actions give rise to interesting spectral triples. This article proceeds with studying the metric induced on state spaces by ergodic actions. More recently, the article [30] produced a detailed construction of a so called Lie-Dirac operator on a $C^{*}$-algebra $A$, based on an ergodic action of a compact Lie group $G$ on $A$. It also investigated the analytic properties of these Lie-Dirac operators, proving in particular that they are finitely summable spectral triples. In the present article, we elaborate on the techniques used in [30] in order to prove quite different results.

Indeed, in [30] the focus was on a Dirac operator for a "noncommutative spin manifold", whereas here the emphasis is on a sort of Hodge-de Rham operator associated with a conformally perturbed metric, construction of twisted spectral triples and the analog of the Chern-GaussBonnet theorem. The Hodge-de Rham operators constructed here are (in general) not Lie-Dirac operators in the sense of [30]. In this previous article, the algebra structure of $A$ played only a minor role in the analytical properties of the spectral triple. Here, the multiplication of $A$ has a central importance.

For a recent approach of Hodge theory using Hilbert modules, we refer to the recent article [44]. See also [42, 43].

This article is organized as follows. In Section 2, we recall the necessary statements from representation theory and operator theory, and the notion of ordinary and twisted spectral triples along with their main properties that are used in our arguments and concern our constructions. We associate a complex of noncommutative differential forms to a $C^{*}$-dynamical system $(A, G, \alpha)$ in Section 3. In the ergodic case, the analog of the Hodge-de Rham operator is studied when the complex is equipped with a Hermitian structure determined by a metric in the conformal class of the standard metric associated with the unique $G$-invariant trace on $A$.

Inspired by a construction in [18], we construct in Section 4 a spectral triple on $A$ and a twisted spectral triple on the opposite algebra $A^{\text {op }}$, which encode the geometric information of the conformally perturbed metric. We study the Dirac operator of the perturbed metric carefully and prove that it is selfadjoint and enjoys having the same spectral dimension as the non-perturbed case. It should be stressed that ergodicity plays a crucial role for the latter to hold.

The existence of an analog of the Chern-Gauss-Bonnet theorem is studied in Section 5 by proving a Hodge decomposition theorem for our complex and showing that its Euler characteristic is independent of the conformal factor. Combining this with the McKean-Singer index formula and small time asymptotic expansions, which often exist for noncommutative geometric spaces, we explain how the analog of the Euler class or the Pfaffian of the curvature form can be computed as local geometric invariants of examples that fit into our setting. Indeed, such invariants depend on the behavior at infinity of the eigenvalues of the involved Laplacians and the action of the algebra. Finally, our main results and conclusions are summarized in Section 6.

\section{Preliminaries}

We start by some reminders about results and notations from various anterior articles.

Definition 2.1. Given a strongly continuous action $\alpha$ of a compact group $G$ on a unital $C^{*}$ algebra $A$, we say that it is ergodic if the fixed algebra of $G$-invariants elements is reduced to the scalars, i.e., if $\forall g \in G, \alpha_{g}(a)=a$, then $a \in \mathbb{C} 1_{A}$.

Among the important results obtained with this notion of ergodic action, let us quote the following [35, Theorem 4.1, p. 82]:

Theorem 2.2. Let $A$ be a unital $C^{*}$-algebra, $G$ a compact group and $\alpha$ a strongly continuous representation of $G$ as an ergodic group of *-automorphisms of $A$, then the unique $G$-invariant state $\varphi_{0}$ on $A$ is a trace. 
Another result that will play an important role in our article is [35, Proposition 2.1, p. 76], which we adapt slightly in the following:

Proposition 2.3. Let $A$ be a unital $C^{*}$-algebra, $G$ a compact group and $\alpha$ a strongly continuous representation of $G$ as an ergodic group of *-automorphisms of $A$. Let $V$ be an irreducible unitary representation of $G, A(V)$ the spectral subspace of $V$ in $A$ and $m(V)$ the multiplicity of $V$ in $A(V)$. Then we have

$$
m(V) \leqslant \operatorname{dim} V \text {. }
$$

Among our main results, we prove the finite summability of certain spectral triples (ordinary and twisted), we therefore define those terms:

Definition 2.4. Let $A$ be a unital $C^{*}$-algebra. An odd (ordinary) spectral triple, also called an odd unbounded Fredholm module, is a triple $(\mathcal{A}, \mathscr{H}, D)$ where

- $\mathscr{H}$ is a Hilbert space and $\pi: A \rightarrow B(\mathscr{H})$ a $*$-representation of $A$ as bounded operators on $\mathscr{H}$

- $D$ is a selfadjoint unbounded operator - which we will call the Dirac operator - with domain $\operatorname{Dom}(D)$,

such that

(i) $\left(1+D^{2}\right)^{-1}$ is a compact operator,

(ii) the subalgebra $\mathcal{A}$ of all $a \in A$ such that

$$
\pi(a)(\operatorname{Dom}(D)) \subseteq \operatorname{Dom}(D) \quad \text { and } \quad[D, \pi(a)] \text { extends to a bounded map on } \mathscr{H}
$$

is dense in $A$.

An even spectral triple is given by the same data, but we further require that a grading $\gamma$ be given on $\mathscr{H}$ such that (i) $A$ acts by even operators, (ii) $D$ is odd.

Remark 2.5. For a selfadjoint operator $D$, condition (i) of the definition above is actually equivalent to $\exists \lambda \in \mathbb{R} \backslash\{0\}$ s.t. $(D+i \lambda)^{-1}$ is a compact operator.

To define finitely summable spectral triples, we now need a brief reminder regarding trace ideals (also known as symmetric ideals), for which we follow Chapter IV of [14]. For more details concerning symmetrically normed operator ideals and singular traces we refer the reader to [53] and $[46]$.

Definition 2.6. For $p>1$, the ideal $\mathscr{L}^{p^{+}}$(also denoted $\mathscr{L}^{(p, \infty)}$ in [14] and $\mathcal{J}_{p, \omega}$ in [53, p. 21]) consists of all compact operators $T$ on $\mathscr{H}$ such that

$$
\|T\|_{p^{+}}:=\sup _{k} \frac{\sigma_{k}(T)}{k^{(p-1) / p}}<\infty
$$

where $\sigma_{k}$ is defined as the supremum of the trace norms of $T E$, when $E$ is an orthonormal projection of dimension $k$, i.e.,

$$
\sigma_{k}(T):=\sup \left\{\|T E\|_{1}, \operatorname{dim} E=k\right\} .
$$

Equivalently, $\sigma_{k}(T)$ is the sum of the $k$ largest eigenvalues (counted with their multiplicities) of the positive compact operator $|T|:=\left(T^{*} T\right)^{1 / 2}$. The definition extends to the case of $p=1$ : $\mathscr{L}^{1^{+}}$is the ideal of compact operators $T$ s.t.

$$
\|T\|_{1^{+}}:=\sup _{k} \frac{\sigma_{k}(T)}{\log k}<\infty .
$$

The elements of $\mathscr{L}^{p^{+}}$are called $p^{+}$-summable (or $(p, \infty)$-summable - see [14, Section IV. $2 \alpha$, p. 299 and following]). 
A spectral dimension for spectral triples is defined as follows

Definition 2.7. A spectral triple is $p^{+}$-summable if $\left(1+D^{2}\right)^{-1 / 2} \in \mathscr{L}^{p^{+}}$.

Finally, we will consider twisted spectral triples (also called $\sigma$-spectral triples) as introduced in [16, Definition 3.1]. This is a spectral triple just like in Definition 2.4, but for a fixed automorphism $\sigma$ of $\mathcal{A}$, the bounded commutators condition (denoted (ii) above) is replaced by

(ii) the subalgebra $\mathcal{A}$ of all $a \in A$ such that

- $\pi(a)(\operatorname{Dom}(D)) \subseteq \operatorname{Dom}(D)$,

- $D \pi(a)-\pi(\sigma(a)) D$ extends to a bounded map on $\mathscr{H}$

is dense in $A$.

In this paper, we will need the subalgebras $\mathcal{A}^{k}$ for $k \geqslant 0$, corresponding to the $C^{k}$-differentiable class. Following [5, Section 2.2], we introduce the space

$$
\mathcal{A}^{m}:=\left\{a \in A: g \mapsto \alpha_{g}(a) \text { is in } C^{m}(G, A)\right\} .
$$

Let us fix a basis $\left(\partial_{i}\right)$ of the Lie algebra $\mathfrak{g}$. For such a choice of basis, the infinitesimal generators $\partial_{i}$ act as derivations $\mathcal{A}^{m} \rightarrow \mathcal{A}^{m-1}$. According to [5, Example 2.2.4, p. 41], $\mathcal{A}^{m}$ equipped with the norm

$$
\|a\|_{m}:=\|a\|+\sum_{k=1}^{m} \sum_{i_{1}=1}^{n} \cdots \sum_{i_{k}=1}^{n} \frac{\left\|\partial_{i_{1}} \cdots \partial_{i_{k}}(a)\right\|}{k !},
$$

is a Banach algebra with $\|a b\|_{m} \leqslant\|a\|_{m}\|b\|_{m}$. In particular, if $h \in \mathcal{A}^{1}$, then $e^{\lambda h} \in \mathcal{A}^{1}$, for all complex number $\lambda$ - see also Lemma 3.3 below for a more precise estimate.

Following the density properties established in [5] (see, e.g., Definition 2.2.15, p. 47), the intersection $\mathcal{A}^{\infty}=\bigcap_{j=0}^{\infty} \mathcal{A}^{j}$ is a dense *-subalgebra of the $C^{*}$-algebra $A$, which is stable under the derivations $\partial_{i}$.

\section{Hodge-de Rham Dirac operator and $C^{*}$-dynamical systems}

In this article, we consider a fixed $A$, a $C^{*}$-algebra with an ergodic action $\alpha$ of a compact Lie group $G$ of dimension $n$. We write $\mathcal{A}$ for $\mathcal{A}^{\infty}$, the "smooth subalgebra" of $A$, which can alternatively be defined as

$$
\mathcal{A}:=\left\{a \in A: g \mapsto \alpha_{g}(a) \text { is in } C^{\infty}(G, A)\right\} .
$$

The Chevalley-Eilenberg cochain complex with coefficients in $\mathcal{A}$ provides a complex that we interpret as "differential forms" on $\mathcal{A}$. For the reader's convenience and to fix notations, we provide a reminder of this construction. For all $k \in \mathbb{N}$,

$$
\Omega^{k}:=\mathcal{A} \otimes \bigwedge^{k} \mathfrak{g}^{*}
$$

where $\mathfrak{g}^{*}$ denotes the linear forms on $\mathfrak{g}$, the Lie algebra of the Lie group $G$. Given a scalar product on $\mathfrak{g}^{*}$ (e.g., obtained from the Killing form), we can extend it to a scalar product on $\bigwedge^{k} \mathfrak{g}^{*}$ by setting

$$
\left\langle v_{1} \wedge \cdots \wedge v_{k}, w_{1} \wedge \cdots \wedge w_{k}\right\rangle:=\operatorname{det}\left(\left\langle v_{i}, w_{j}\right\rangle\right),
$$


i.e., the determinant of the matrix of scalar products. We fix an orthonormal basis $\left(\omega_{j}\right)_{j=1, \ldots, n}$ of $\mathfrak{g}^{*}$ for this scalar product and consider its dual basis $\left(\partial_{j}\right)_{j=1, \ldots, n}$ in $\mathfrak{g}$.

Following [41, the model of (4.6), p. 157], we write the exterior derivative of the complex

$$
\begin{aligned}
d(a \otimes & \left.\omega_{i_{1}} \wedge \omega_{i_{2}} \wedge \cdots \wedge \omega_{i_{K}}\right)=\sum_{j=1}^{n} \partial_{j}(a) \otimes \omega_{j} \wedge \omega_{i_{1}} \wedge \omega_{i_{2}} \wedge \cdots \wedge \omega_{i_{K}} \\
& -\frac{1}{2} \sum_{k=1}^{K} \sum_{\alpha, \beta}(-1)^{k+1} c_{\alpha \beta}^{i_{k}} a \otimes \omega_{\alpha} \wedge \omega_{\beta} \wedge \omega_{i_{1}} \wedge \cdots \wedge \omega_{i_{k-1}} \wedge \omega_{i_{k+1}} \wedge \cdots \wedge \omega_{i_{K}},
\end{aligned}
$$

where $\left[\partial_{i}, \partial_{j}\right]=\sum_{k=1}^{n} c_{i j}^{k} \partial_{k}-$ the $c_{i j}^{k}$ are called the structure constants of the Lie algebra $\mathfrak{g}$. A lengthy but straightforward computation proves that this exterior derivative satisfies $d^{2}=0$ on $\Omega^{\bullet}$, therefore $\left(\Omega^{\bullet}, d\right)$ is a complex.

Remark 3.1. The Chevalley-Eilenberg complex is available even for noncompact groups $G$ and nonergodic actions. In other words, the square $d^{2}$ actually vanishes even when $G$ is not a compact Lie group and when the action of $G$ on $A$ is not ergodic.

The natural product on $\Omega^{\bullet}$ is

$$
\left(a \otimes v_{1} \wedge \cdots \wedge v_{k}\right) \cdot\left(a^{\prime} \otimes w_{1} \wedge \cdots \wedge w_{k^{\prime}}\right):=a a^{\prime} \otimes v_{1} \wedge \cdots \wedge v_{k} \wedge w_{1} \wedge \cdots \wedge w_{k^{\prime}}
$$

i.e., the product of a $k$-form with a $k^{\prime}$-form is a $k+k^{\prime}$-form. In particular, for all $k, \Omega^{k}$ is an $\mathcal{A}$-bimodule. The exterior derivative $d$ is compatible with the right module structure in the following sense

$$
\begin{aligned}
d\left(a a^{\prime} \otimes\right. & \left.\omega_{i_{1}} \wedge \omega_{i_{2}} \wedge \cdots \wedge \omega_{i_{K}}\right)=\sum_{j=1}^{n} \partial_{j}\left(a a^{\prime}\right) \otimes \omega_{j} \wedge \omega_{i_{1}} \wedge \omega_{i_{2}} \wedge \cdots \wedge \omega_{i_{K}} \\
& -\frac{1}{2} \sum_{k} \sum_{\alpha, \beta} c_{\alpha \beta}^{i_{k}} a a^{\prime} \otimes \omega_{\alpha} \wedge \omega_{\beta} \wedge \omega_{i_{1}} \wedge \cdots \wedge \omega_{i_{k-1}} \wedge \omega_{i_{k+1}} \wedge \cdots \wedge \omega_{i_{K}} \\
= & \left(\sum_{j=1}^{n} \partial_{j}(a) \otimes \omega_{j} \wedge \omega_{i_{1}} \wedge \omega_{i_{2}} \wedge \cdots \wedge \omega_{i_{K}}\right) a^{\prime} \\
& +\sum_{j=1}^{n} a \partial_{j}\left(a^{\prime}\right) \otimes \omega_{j} \wedge \omega_{i_{1}} \wedge \omega_{i_{2}} \wedge \cdots \wedge \omega_{i_{K}} \\
& -\frac{1}{2}\left(\sum_{k} \sum_{\alpha, \beta} c_{\alpha \beta}^{i_{k}} a \otimes \omega_{\alpha} \wedge \omega_{\beta} \wedge \omega_{i_{1}} \wedge \cdots \wedge \omega_{i_{k-1}} \wedge \omega_{i_{k+1}} \wedge \cdots \wedge \omega_{i_{K}}\right) a^{\prime} \\
= & d\left(a \otimes \omega_{i_{1}} \wedge \omega_{i_{2}} \wedge \cdots \wedge \omega_{i_{K}}\right) a^{\prime}+(-1)^{K}\left(a \otimes \omega_{j} \wedge \omega_{i_{1}} \wedge \omega_{i_{2}} \wedge \cdots \wedge \omega_{i_{K}}\right) d\left(a^{\prime}\right) .
\end{aligned}
$$

Since we want to treat conformal deformations of the original structure, we follow [18] and fix a positive invertible element $e^{h} \in \mathcal{A}^{1}$, where $h$ is a smooth selfadjoint element in $\mathcal{A}^{1}$. Then we define a scalar product on $\Omega^{k}$ by the formula

$$
\left(a \otimes v_{1} \wedge \cdots \wedge v_{k}, a^{\prime} \otimes w_{1} \wedge \cdots \wedge w_{k}\right)_{\varphi}:=\varphi_{0}\left(a^{*} a^{\prime} e^{(n / 2-k) h}\right) \operatorname{det}\left(\left\langle v_{i}, w_{j}\right\rangle\right),
$$

where $\varphi_{0}$ is the unique $G$-invariant state on $A$, which is actually a trace according to $[1$, Theorem 3.1, p. 8]. We set the scalar product of two forms of different degrees to vanish. The scalar product obtained for $h=0$ is the one we call the natural scalar product on forms. We define 
the Hilbert space $\mathscr{H}_{\varphi}$ as the completion of $\Omega^{\bullet}$ for the scalar product (3.3). In the particular case of $h=0$, we obtain our reference Hilbert space $\mathscr{H}$. We will also need the Hilbert spaces $\mathscr{H}_{0, \varphi}:=\operatorname{GNS}(\mathcal{A}, \varphi)$ and $\mathscr{H}_{0}:=\operatorname{GNS}\left(\mathcal{A}, \varphi_{0}\right)$ as well as the Hilbert spaces $\mathscr{H}_{k}:=\mathscr{H}_{0} \otimes \bigwedge^{k} \mathfrak{g}^{*}-$ i.e., the completion of $k$-forms - and $\mathscr{H}_{k, \varphi}$.

To understand why we choose the form (3.3) for the conformal deformation, we compare with the commutative case of a $n$-dimensional compact manifold $M$, where we have the following property: if the Riemannian metric is transformed by $g \rightsquigarrow \lambda g$ (for $\lambda>0$ ), then the (pointwise) norm of all vectors is multiplied by $\lambda^{1 / 2}$ and thus the pointwise norm of 1 -forms is multiplied by $\lambda^{-1 / 2}$. This in turn implies that the pointwise norm of $k$-form is multiplied by $\lambda^{-k / 2}$. Finally, the (global) scalar product of $k$-forms is the integral of the pointwise scalar products. Since under the conformal deformation, the total volume of the manifold $M$ is multiplied by $\lambda^{n / 2}$, the (global) scalar products of $k$-forms are multiplied by $\lambda^{n / 2-k}$. In particular, if $n$ is even and $k=n / 2$, then the scalar product on $n / 2$-forms is left invariant under the conformal deformation.

In order to study $d$ and its adjoint, we introduce the degree 1 maps $T_{j}: \bigwedge^{\bullet} \mathfrak{g}^{*} \rightarrow \bigwedge^{\bullet} \mathfrak{g}^{*}$ defined for all $j \in\{1, \ldots, n\}$ by

$$
T_{j}\left(v_{1} \wedge \cdots \wedge v_{k}\right)=\omega_{j} \wedge v_{1} \wedge \cdots \wedge v_{k} .
$$

Let $\mathcal{R}_{x}$ denote the right multiplication operator for any $x \in A: \mathcal{R}_{x}(a)=a x$, and let $B_{\alpha \beta}^{i_{k}}$ be the bounded operator on $\bigwedge^{\bullet} \mathfrak{g}^{*}$ defined using the basis $\left(\omega_{j}\right)$ by

$$
B_{\alpha \beta}^{i_{k}}\left(\omega_{i_{1}} \wedge \omega_{i_{2}} \wedge \cdots \wedge \omega_{i_{K}}\right)=\omega_{\alpha} \wedge \omega_{\beta} \wedge \omega_{i_{1}} \wedge \cdots \wedge \omega_{i_{k-1}} \wedge \omega_{i_{k+1}} \wedge \cdots \wedge \omega_{i_{K}} .
$$

We can now give an explicit form to the operator $d$ and its (formal) adjoint for the unperturbed metric.

Lemma 3.2. With the previous notations, when $h=0$, the operator $d$ can be written

$$
d=\sum_{j} \partial_{j} \otimes T_{j}-\frac{1}{2} \sum_{k, \alpha, \beta} c_{\alpha \beta}^{i_{k}} \otimes B_{\alpha \beta}^{i_{k}}
$$

and its adjoint $d^{*}$ is

$$
d^{*}=\sum_{j} \partial_{j} \otimes T_{j}^{*}-\frac{1}{2} \sum_{k, \alpha, \beta} \overline{c_{\alpha \beta}^{i_{k}}} \otimes\left(B_{\alpha \beta}^{i_{k}}\right)^{*} .
$$

Proof. The only point that is not self-explanatory is the behavior of $\partial_{j}$ with respect to the trace $\varphi_{0}$

$$
\left\langle\left[\partial_{j}(a)\right],\left[a^{\prime}\right]\right\rangle=\varphi_{0}\left(\partial_{j}(a)^{*} a^{\prime}\right)=\varphi_{0}\left(-\partial_{j}\left(a^{*}\right) a^{\prime}\right)=\varphi_{0}\left(a^{*} \partial_{j}\left(a^{\prime}\right)\right),
$$

where we used the relations $\partial_{j}(a)^{*}=-\partial_{j}\left(a^{*}\right)$ and $\varphi_{0}\left(\partial_{j}(a)\right)=0$.

Lemma 3.3. Let $h$ be an element of $\mathcal{A}^{1}$ and $\partial$ be an infinitesimal generator of $G$, acting as a derivation on $\mathcal{A}^{1}$, then $\partial\left(e^{h}\right)$ is in the $C^{*}$-algebra $A$ and satisfies

$$
\left\|\partial\left(e^{h}\right)\right\| \leqslant\|\partial(h)\| e^{\|h\|} .
$$

In particular, for a scalar parameter $v \rightarrow 0, \partial\left(e^{v h}\right) \rightarrow 0$.

Proof. As an operator from $\mathcal{A}^{1}$ to $A$, the derivation $\partial$ is continuous, therefore we can estimate $\partial\left(e^{h}\right)$ by using an $\mathcal{A}^{1}$-converging sequence, like the partial sums of $e^{h}$.

For this sequence, using the derivation property, we get

$$
\left\|\partial\left(\sum_{k=0}^{N} \frac{h^{k}}{k !}\right)\right\| \leqslant \sum_{k=1}^{N} k \frac{\|\partial h\|}{k} \frac{\|h\|^{k-1}}{(k-1) !} \leqslant\|\partial h\| e^{\|h\|} .
$$

The property $\partial\left(e^{v h}\right) \rightarrow 0$ as $v \rightarrow 0$ follows immediately. 
We call $d_{\varphi}$ the operator defined on $\mathscr{H}_{\varphi}$ by the formula (3.1). Once the scalar product (3.3) is defined, we want to define an adjoint $d_{\varphi}^{*}$ to $d_{\varphi}$ for this scalar product. The Hodge-de Rham operator that we would like to study in fine is $d_{\varphi}+d_{\varphi}^{*}$. However, the unbounded operator $d_{\varphi}$ is a priori arbitrary, so it is not clear that it admits a densely defined adjoint. To clarify the relations between $\mathscr{H}$ and $\mathscr{H}_{\varphi}$, we introduce the following lemma:

Lemma 3.4. For any selfadjoint $h \in \mathcal{A}^{1}$,

- the Hilbert space $\mathscr{H}_{\varphi}$ is equipped with a $G$-representation defined on degree $k$ forms by $\mathbb{V}_{g}\left(\left[a \otimes v_{1} \wedge \cdots \wedge v_{k}\right]_{\varphi}\right)=\left[\alpha_{g}(a) \otimes v_{1} \wedge \cdots \wedge v_{k}\right]_{\varphi}$, which leads to a G-equivariant left $\mathcal{A}$-module structures on $\mathscr{H}_{\varphi}$;

- the map $L: \mathscr{H}_{0} \rightarrow \mathscr{H}_{0, \varphi}$ defined by $L([a]):=[a]_{\varphi}$ is invertible and intertwines the $G$ equivariant left $\mathcal{A}$-module structures on $\mathscr{H}_{0}$ and $\mathscr{H}_{0, \varphi}$.

Of course, $L$ extends to $L \otimes \mathrm{Id}: \mathscr{H} \rightarrow \mathscr{H}_{\varphi}$ which is still a continuous and invertible map. We denote its adjoint by $H: \mathscr{H}_{\varphi} \rightarrow \mathscr{H}$, whose explicit form is

$$
H\left(\left[a \otimes v_{1} \wedge \cdots \wedge v_{k}\right]_{\varphi}\right)=\left[a e^{(n / 2-k) h} \otimes v_{1} \wedge \cdots \wedge v_{k}\right] .
$$

Finally, $\mathscr{H}$ and $\mathscr{H}_{\varphi}$ are related by the unitary map $U: \mathscr{H} \rightarrow \mathscr{H}_{\varphi}$ given on degree $k$ forms by

$$
U\left(\left[a \otimes v_{1} \wedge \cdots \wedge v_{k}\right]\right)=\left[a e^{-(n / 2-k) h / 2} \otimes v_{1} \wedge \cdots \wedge v_{k}\right]_{\varphi}
$$

Remark 3.5. The map $U$ defined above is unitary, but it does not intertwine the $G$-structures on $\mathscr{H}$ and $\mathscr{H}_{\varphi}$.

Proof. Since the sum $\mathscr{H}_{\varphi}=\bigoplus_{k} \mathscr{H}_{k, \varphi}$ is finite, it suffices to check that $\mathbb{V}_{g}$ is continuous on each $\mathscr{H}_{k, \varphi}$ separately. Since $\mathbb{V}_{g}$ does not act on $\Lambda^{\bullet} \mathfrak{g}^{*}$, it is enough to prove continuity on $\operatorname{GNS}(A, \tilde{\varphi})$ where $\tilde{\varphi}(a)=\varphi_{0}\left(a e^{-h_{k}}\right)$ and $h_{k}=-(n / 2-k) h \in \mathcal{A}^{1}$ (corresponding to forms of degree $k)$. We get

$$
\begin{aligned}
\left\|\alpha_{g}(a)\right\|_{\tilde{\varphi}}^{2} & =\varphi_{0}\left(\alpha_{g}(a)^{*} \alpha_{g}(a) e^{-h_{k}}\right)=\varphi_{0}\left(a^{*} a \alpha_{g^{-1}}\left(e^{-h_{k}}\right)\right) \\
& =\varphi_{0}\left(a e^{-h_{k} / 2} e^{h_{k} / 2} \alpha_{g^{-1}}\left(e^{-h_{k}}\right) e^{h_{k} / 2} e^{-h_{k} / 2} a^{*}\right) \leqslant K \varphi_{0}\left(a e^{-h_{k}} a^{*}\right)=K\|a\|_{\tilde{\varphi}}^{2},
\end{aligned}
$$

for a constant $K=\left\|e^{h_{k} / 2} \alpha_{g^{-1}}\left(e^{-h_{k}}\right) e^{h_{k} / 2}\right\|$. The above (scalar) inequality follows from the inequality of operators

$$
a e^{-h_{k} / 2} e^{h_{k} / 2} \alpha_{g^{-1}}\left(e^{-h_{k}}\right) e^{h_{k} / 2} e^{-h_{k} / 2} a^{*} \leqslant a e^{-h_{k} / 2} K e^{-h_{k} / 2} a^{*}=K a e^{-h_{k}} a^{*},
$$

which is valid since $e^{h_{k} / 2} \alpha_{g^{-1}}\left(e^{-h_{k}}\right) e^{h_{k} / 2}$ is a positive operator. It then suffices to apply the positive functional $\varphi_{0}$. Once we know that the map $\mathbb{V}_{g}$ is defined on the full Hilbert space, proving that it is compatible with the left $\mathcal{A}$-module structure is a formality.

The process is similar for $L$ : it is clear from the definition that if the map $L$ exists, then it intertwines the $G$-equivariant left $\mathcal{A}$-module structures on $\mathscr{H}$ and $\mathscr{H}_{\varphi}$. It remains to prove that $L$ is well-defined and invertible.

We first evaluate

$$
\|L(a)\|_{\tilde{\varphi}}^{2}=\varphi_{0}\left(a^{*} a e^{-h_{k}}\right)=\varphi_{0}\left(a e^{-h_{k}} a^{*}\right) \leqslant\left\|e^{-h_{k}}\right\| \varphi_{0}\left(a a^{*}\right)=\left\|e^{-h_{k}}\right\|\|a\|_{\varphi_{0}}^{2},
$$

by the same argument as above.

To prove that $L$ is invertible, consider the norm of its inverse

$$
\|a\|_{\varphi_{0}}^{2}=\varphi_{0}\left(a e^{-h_{k} / 2} e^{h_{k}} e^{-h_{k} / 2} a^{*}\right) \leqslant\left\|e^{h_{k}}\right\| \varphi_{0}\left(a e^{-h_{k}} a^{*}\right)=\left\|e^{h_{k}}\right\|\|a\|_{\tilde{\varphi}}^{2} .
$$

The evaluation of the adjoint $H$ of $L$ and of the unitary map $U: \mathscr{H} \rightarrow \mathscr{H}_{\varphi}$ is an easy exercise. 
With the previous notations, we see that $d_{\varphi}=(L \otimes \mathrm{Id}) d(L \otimes \mathrm{Id})^{-1}$ and thus (at least formally) $d_{\varphi}^{*}=H^{-1} d^{*} H$. However, in order to facilitate the comparison between $d_{\varphi}+d_{\varphi}^{*}$ acting on $\mathscr{H}_{\varphi}$ and $d+d^{*}$ acting on $\mathscr{H}$, we "push" $d_{\varphi}+d_{\varphi}^{*}$ to $\mathscr{H}$ using the unitary $U$. This leads us to the operators $D_{u}$ studied in the Proposition 3.6 below. But first, for $h=h^{*}$, we need to introduce the operators $K_{u}: \mathscr{H} \rightarrow \mathscr{H}$, where $u \in \mathbb{R}$, defined by

$$
K_{u}\left(\left[a \otimes v_{1} \wedge \cdots \wedge v_{k}\right]\right)=\left[a e^{(n / 2-k) h u} \otimes v_{1} \wedge \cdots \wedge v_{k}\right] .
$$

This is a one-parameter group of invertible selfadjoint operators. Moreover, following our remark on $\mathcal{A}^{1}$ at the end of Section 2 , for all $u \in \mathbb{R}$, the operators $K_{u}$ preserve the space $\mathcal{A}^{1} \otimes \Lambda^{\bullet} \mathfrak{g}^{*}$.

In the proof below, we consider the orthogonal projections $\Pi_{k}: \mathscr{H} \rightarrow \mathscr{H}_{k}$ onto the completion of the $k$-forms, for all $k \in\{0, \ldots, n\}$.

Proposition 3.6. For all $u \in[0,1]$, we consider two unbounded operators defined on the dense domain $C=\mathcal{A}^{1} \otimes \bigwedge^{\bullet} \mathfrak{g}^{*} \subseteq \mathscr{H}$,

$$
d_{u}:=K_{u} d K_{-u}, \quad d_{u}^{*}:=K_{-u} d^{*} K_{u}
$$

We have:

(1) if $E_{+, u}$ and $E_{-, u}$ are, respectively, the closures in $\mathscr{H}$ of the images of the operators $d_{u}$ and $d_{u}^{*}$, then $E_{+, u}$ and $E_{-, u}$ are orthogonal in $\mathscr{H}$; we denote by $\Pi_{+, u}$ and $\Pi_{-, u}$, respectively, the orthogonal projections on these spaces;

(2) the operator

$$
D_{u}=K_{u} d K_{-u}+K_{-u} d^{*} K_{u},
$$

is essentially selfadjoint on a common core domain $C=\mathcal{A}^{1} \otimes \bigwedge^{\bullet} \mathfrak{g}^{*}$.

The family of operators $D_{u}$ satisfies the estimate

$$
\left\|\left(D_{u+v}-D_{u}\right)(\underline{\omega})\right\| \leqslant o_{v}(1)\left\|D_{u} \underline{\omega}\right\|+o_{v}(1)\|\underline{\omega}\|,
$$

where $\underline{\omega}$ is any vector in the common selfadjointness domain and following Landau's notations, $o_{v}(1)$ stands for functions of $v$ which tend to 0 for $v \rightarrow 0$. We can choose these two functions independently of the parameter $u \in[0,1]$.

Proof. Regarding point (1), we start by proving the property for $u=0$, i.e., for the untwisted case. There, following Lemma 3.4 the trace $\varphi_{0}$ is $G$-invariant and therefore the action $\mathbb{V}_{g}$ of $G$ on $\mathscr{H}_{0}$ is unitary. Consequently, the $G$-representation can be decomposed into a direct sum of finite-dimensional $G$-representations. Let us denote by $V$ one of these finite-dimensional spaces.

It is clear from the definition (3.1) that both $V \otimes \bigwedge^{k} \mathfrak{g}^{*} \subseteq \mathscr{H}$ and its orthogonal are stable under the action of $d$. Thus the restriction of $d$ to the finite-dimensional space $V \otimes \bigwedge^{k} \mathfrak{g}^{*}$ is bounded and admits an adjoint $d^{*}$ whose form is given by Lemma 3.2. Varying the space $V$, we see that $d^{*}$ is defined on $\mathscr{D}$, the algebraic direct sum of $V \otimes \Lambda^{k} \mathfrak{g}^{*}$, which is a dense subset of $\mathscr{H}$. If we restrict to the case of forms $\underline{\omega}, \underline{\omega}^{\prime}$ in the space $V \otimes \bigwedge^{k} \mathfrak{g}^{*} \subseteq \mathscr{H}$, we have

$$
\left\langle d \underline{\omega}, d^{*} \underline{\omega}^{\prime}\right\rangle=\left\langle d^{2} \underline{\omega}, \underline{\omega}^{\prime}\right\rangle=0
$$

there are no considerations of domains for $d$ and $d^{*}$, since we consider finite-dimensional spaces. The same argument applied to different finite vector spaces $V$ proves that $E_{+, 0}$ (the image of $d$ ) and $E_{-, 0}\left(\right.$ the image of $\left.d^{*}\right)$ are orthogonal. 
To treat point (1) for a general $u \in[0,1]$, we note that $E_{+, u}=K_{u} E_{+, 0}$ : indeed, if $\xi=$ $\lim d \underline{\omega}_{n}$, then $K_{u} \xi=\lim K_{u} d K_{-u}\left(K_{u} \underline{\omega}_{n}\right)$ and vice versa. Similarly, $E_{-, u}=K_{-u} E_{-, 0}$. Given $K_{u} e_{+} \in E_{+, u}$ and $K_{-u} e_{-} \in E_{-, u}$, we have

$$
\left\langle K_{u} e_{+}, K_{-u} e_{-}\right\rangle=\left\langle e_{+}, e_{-}\right\rangle=0,
$$

since $K_{u}$ is selfadjoint and $e_{+} \in E_{+, 0}, e_{-} \in E_{-, 0}$ are orthogonal. This proves the requested orthogonality relation.

Regarding point (2), let us start by giving a sketch of the proof: we first prove that $D_{0}=D$ is essentially selfadjoint on the requested domain. Using the estimate (3.5), we then apply Kato-Rellich theorem to show that if $D_{u}$ is essentially selfadjoint for the domain $C$, then so is the operator $D_{u+v}$ for all $|v| \leqslant \varepsilon$, where $\varepsilon$ is independent of the point $u \in[0,1]$ chosen. As a consequence, all operators $D_{u}$ are essentially selfadjoint for the fixed domain.

We first prove that $D_{0}$ is selfadjoint. This is done by using the Peter-Weyl decomposition of $\mathscr{H}_{0}$ for the unitary action $\mathbb{V}_{g}$ of $G$ on $\mathscr{H}_{0}$. As mentioned in point (1), the restriction of $d$ to this finite-dimensional space $V \otimes \bigwedge^{k} \mathfrak{g}^{*} \subseteq \mathscr{H}$ is well-defined, as is its adjoint $d^{*}$. Varying the space $V$, we consider $\mathscr{D}$, the direct sum of $V \otimes \bigwedge^{k} \mathfrak{g}^{*}$, which is a dense subset of $\mathscr{H}$.

In this situation, we can define $D=d+d^{*}$ on $\mathscr{D}$. If we restrict $D$ to a component $V \otimes$ $\bigwedge^{k} \mathfrak{g}^{*} \subseteq \mathscr{H}$, it is formally selfadjoint by definition. It therefore admits an orthonormal basis of eigenvectors with real associated eigenvalues. It follows that $\operatorname{Ran}(D+i)$ and $\operatorname{Ran}(D-i)$ are dense in $\mathscr{H}$, and this is enough to prove that $D$ is essentially selfadjoint on the domain $C$ (see [50, Corollary, p. 257]).

Let us now consider an arbitrary $u \in[0,1]$. We want to find $\varepsilon>0$ uniform in $u$ and small enough so that for all $v$ with $|v| \leqslant \varepsilon$, the operator $D_{u+v}$ is essentially selfadjoint. By definition,

$$
D_{u+v}=K_{u+v} d K_{-(u+v)}+K_{-(u+v)} d^{*} K_{u+v}
$$

If we introduce $R_{v}=K_{v}-1$, then we can write

$$
K_{u+v}=K_{u}\left(1+R_{v}\right), \quad K_{-(u+v)}=\left(1+R_{-v}\right) K_{-u} .
$$

It is clear that both $R_{v}$ and $R_{-v}$ are bounded with $\left\|R_{v}\right\| \rightarrow 0,\left\|R_{-v}\right\| \rightarrow 0$ for $v \rightarrow 0$ and by definition, for all $v \in \mathbb{R}, R_{v}$ commutes with $K_{u}$ for $u \in \mathbb{R}$.

We write

$$
\begin{aligned}
D_{u+v}= & K_{u}\left(1+R_{v}\right) d\left(1+R_{-v}\right) K_{-u}+K_{-u}\left(1+R_{-v}\right) d^{*}\left(1+R_{v}\right) K_{u} \\
= & K_{u} d K_{-u}+K_{u} d R_{-v} K_{-u}+K_{u} R_{v} d K_{-u}+K_{u} R_{v} d R_{-v} K_{-u} \\
& +K_{-u} d^{*} K_{u}+K_{-u} d^{*} R_{v} K_{u}+K_{-u} R_{-v} d^{*} K_{u}+K_{-u} R_{-v} d^{*} R_{v} K_{u} .
\end{aligned}
$$

The sum of the terms $K_{u} d K_{-u}$ and $K_{-u} d^{*} K_{u}$ gives back $D_{u}$. Since $E_{+, u}$ and $E_{-, u}$ are orthogonal, we have

$$
\left\|D_{u} \underline{\underline{\omega}}\right\|^{2}=\left\|\Pi_{+, u}\left(D_{u} \underline{\underline{\omega}}\right)\right\|^{2}+\left\|\Pi_{-, u}\left(D_{u} \underline{\underline{\omega}}\right)\right\|^{2}=\left\|d_{u} \underline{\underline{\omega}}\right\|^{2}+\left\|d_{u}^{*} \underline{\underline{\omega}}\right\|^{2} .
$$

Both $D_{u+v}$ and $D_{u}$ are symmetric operators, so their difference (namely the sum $\Sigma$ of the six remaining terms) is a symmetric operator. By hypothesis, $D_{u}$ is selfadjoint. According to Kato-Rellich theorem as stated in [51, Theorem X.12, p. 162], it therefore only remains to prove that $C$ is also a domain for $\Sigma$ and that for all $\underline{\omega} \in C$,

$$
\|\Sigma(\underline{\omega})\| \leqslant a\left\|D_{u} \underline{\omega}\right\|+b\|\underline{\omega}\|
$$

where both real numbers $a, b$ are positive and $a<1$. It is clear from the definition of $K_{ \pm u}$ and $R_{v}$ that their actions preserve the core $C$ of $C^{1}$-functions on $G$ and thus $\Sigma(\underline{\omega})$ has a welldefined meaning for all $\underline{\omega} \in C$. We decompose $\underline{\omega} \in C$ into a sum $\underline{\omega}=\sum_{k} \underline{\omega}_{k}$ of $C^{1}$-forms of degree $k$ and start by an estimate of the different terms $\left\|\Sigma \underline{\omega}_{k}\right\|$ for any fixed $k$. 
Remember from Lemma 3.2 that $d$ can be written

$$
d=\sum_{j} \partial_{j} \otimes T_{j}-\frac{1}{2} \sum_{k, \alpha, \beta} c_{\alpha \beta}^{i_{k}} \otimes B_{\alpha \beta}^{i_{k}},
$$

where the different $B_{\alpha \beta}^{i_{k}}$ are bounded operators. We remark that the $B_{\alpha \beta}^{i_{k}}$ commute with right multiplications, like the one appearing in the definition of $K_{u}$ acting on an element of given degree. For $\underline{\omega}=a \otimes \underline{v}$ of degree $k$, we write

$$
\begin{aligned}
K_{u} d R_{-v} K_{-u} a \otimes \underline{v}= & \sum \partial_{j}\left(a e^{-(n / 2-k) h u}\left(e^{-(n / 2-k) h v}-1\right)\right) e^{(n / 2-(k+1)) h u} \otimes T_{j}(\underline{v}) \\
& -\frac{1}{2} \sum_{k} \sum_{\alpha, \beta} c_{\alpha \beta}^{i_{k}} a e^{-(n / 2-k) h u}\left(e^{-(n / 2-k) h v}-1\right) e^{(n / 2-(k+1)) h u} \otimes B_{\alpha \beta}^{i_{k}} \underline{v} \\
= & \sum \partial_{j}\left(a e^{-(n / 2-k) h u}\right) e^{(n / 2-(k+1)) h u}\left(e^{-(n / 2-k) h v}-1\right) \otimes T_{j}(\underline{v}) \\
& -\frac{1}{2} \sum_{k} \sum_{\alpha, \beta} c_{\alpha \beta}^{i_{k}} a e^{-h u}\left(e^{-(n / 2-k) h v}-1\right) \otimes B_{\alpha \beta}^{i_{k}} \underline{v} \\
& \left.+\sum a e^{-(n / 2-k) h u} \partial_{j}\left(e^{-(n / 2-k) h v}-1\right)\right) e^{(n / 2-(k+1)) h u} \otimes T_{j}(\underline{v}) \\
= & R_{-v}\left(K_{u} d K_{-u}\right)(a \otimes \underline{\omega})+K_{u} \circ \sum_{j}\left(\mathcal{R}_{\left.\partial_{j}\left(e^{-(n / 2-k) h v}\right) \otimes T_{j}\right) \circ K_{-u}(a \otimes \underline{v}) .}\right.
\end{aligned}
$$

Taking a linear combination to treat the case of a sum $\underline{\omega}=\sum \underline{\omega}_{k}$, we get

$$
K_{u} d R_{-v} K_{-u}=R_{-v}\left(K_{u} d K_{-u}\right)+K_{u} \circ\left(\sum_{j, k}\left(\mathcal{R}_{\partial_{j}\left(e^{-(n / 2-k) h v}\right)} \otimes T_{j}\right) \circ \Pi_{k}\right) \circ K_{-u}
$$

In this equality, $\sum_{j, k}\left(\mathcal{R}_{\partial_{j}\left(e^{-(n / 2-k) h v}\right)} \otimes T_{j}\right) \circ \Pi_{k}$ is a finite sum of bounded operators. As a consequence of Lemma 3.3, the norm of these operators tend to 0 for $v \rightarrow 0$. We already know that $R_{-v}$ tends to 0 in norm for $v \rightarrow 0$, we therefore get the estimate

$$
\left\|K_{u} d R_{-v} K_{-u}(\underline{\omega})\right\| \leqslant o_{v}(1)\left\|K_{u} d K_{-u}(\underline{\omega})\right\|+o_{v}(1)\|\underline{\omega}\| .
$$

The two functions $o_{v}(1)$ can be taken uniform in $u \in[0,1]$, since $[0,1]$ is a compact.

The term $K_{u} R_{v} d K_{-u}$ is easily treated: $K_{u} R_{v} d K_{-u}=R_{v} K_{u} d K_{-u}$. The term $K_{u} R_{v} d R_{-v} K_{-u}$ is processed similarly: $K_{u} R_{v} d R_{-v} K_{-u}=R_{v} K_{u} d R_{-v} K_{-u}$ and then the estimate (3.7) enables us to write

$$
\left\|K_{u} R_{v} d R_{-v} K_{-u}(\underline{\omega})\right\| \leqslant o_{v}(1)\left\|\left(K_{u} d K_{u}^{-1}\right)(\underline{\omega})\right\|+o_{v}(1)\|\underline{\omega}\| .
$$

As a result, we get

$$
\left\|\left(d_{u+v}-d_{u}\right) \underline{\omega}\right\| \leqslant o_{v}(1)\left\|d_{u} \underline{\omega}\right\|+o_{v}(1)\|\underline{\omega}\| .
$$

Lemma 3.2 affords a similar treatment of the term $K_{-u} d^{*} R_{v} K_{u}$, just replacing $T_{j}$ by $T_{j}^{*}, B_{\alpha \beta}^{i_{k}}$ by $\left(B_{\alpha \beta}^{i_{k}}\right)^{*}$ and $c_{\alpha \beta}^{i_{k}}$ by $\overline{c_{\alpha \beta}^{i_{k}}}$. We get an estimate

$$
\left\|\left(d_{u+v}^{*}-d_{u}^{*}\right) \underline{\omega}\right\| \leqslant o_{v}(1)\left\|d_{u}^{*} \underline{\omega}\right\|+o_{v}(1)\|\underline{\omega}\| .
$$

Using the equation (3.6), which ensures that $\left\|d_{u} \underline{\omega}\right\| \leqslant\left\|D_{u} \underline{\omega}\right\|$ and $\left\|d_{u}^{*} \underline{\omega}\right\| \leqslant\left\|D_{u} \underline{\omega}\right\|$, we can combine (3.8) and (3.9) to show that the relation (3.5) is satisfied.

We can therefore apply the Kato-Rellich theorem for all $u \in[0,1]$ and this proves that all $D_{u}$ (including $D_{1}$ ) have selfadjoint extensions with the same core $C=\mathcal{A}^{1} \otimes \bigwedge^{\bullet} \mathfrak{g}^{*}$. 
Remark 3.7. It appears from the proof of point (2) that we could also take $\mathcal{A}^{\infty} \otimes \bigwedge^{\bullet} \mathfrak{g}^{*}$ as core for the operator $D_{0}$ (using the Peter-Weyl decomposition). If we further assume $h \in \mathcal{A}^{\infty}$, the rest of the proof applies verbatim and shows that all $D_{u}$ have a common core, namely $\mathcal{A}^{\infty} \otimes \bigwedge^{\bullet} \mathfrak{g}^{*}$.

Corollary 3.8. For all selfadjoint elements $h \in \mathcal{A}^{1}$ and all parameters $u \in[0,1]$, the operators $D_{u}$ are $n^{+}$-summable.

Proof. In the untwisted case, i.e., for $D_{0}$, we can follow the argument of Theorem 5.5 of [30] to prove that $d+d^{*}$ is $n^{+}$-summable, where $n$ is the dimension of $G$. Indeed, according to Proposition 2.3 from [35], as $G$-vector spaces, we have $\mathscr{H} \hookrightarrow \mathscr{H}_{\text {ref }}$ where $\mathscr{H}_{\text {ref }}:=L^{2}(G) \otimes \bigwedge^{k} \mathfrak{g}^{*}$. Moreover, the operator $d+d^{*}:=D_{\text {ref }}$ on this space is just the Hodge-de Rham operator on $G$ and therefore it is $n^{+}$-summable. Since $D_{\text {ref }}$ also preserves the finite-dimensional spaces $V \otimes \bigwedge^{k} \mathfrak{g}^{*}$ obtained by Peter-Weyl decomposition, the eigenvalues of $|D|$ coincide with those of $\left|D_{\text {ref }}\right|$ except that they may have lower (and possibly zero) multiplicities. Consequently, the same computation as in [30] proves that $D$ is $n^{+}$-summable.

To extend this property to all $D_{u}$ for $u \in[0,1]$, we first note that to prove $D_{u}$ is $n^{+}$-summable, it suffices to show that the operator $\left(D_{u}+i\right)^{-1}$ is in the symmetric ideal $\mathscr{L}^{n^{+}}-$as mentioned in Remark 2.5. The existence of the operator $\left(D_{u}+i\right)^{-1}$ is a consequence of Proposition 3.6. The discussion above proves that $\left(D_{0}+i\right)^{-1}$ is in this ideal.

We then use [39, Theorem 1.16, p. 196] to prove that if $\left(D_{u}+i\right)^{-1} \in \mathscr{L}^{n^{+}}$then for some $\varepsilon>0$ small enough but independent of $u \in[0,1]$, and for any $v$ in $|v| \leqslant \varepsilon$, then $\left(D_{u+v}+i\right)^{-1} \in \mathscr{L}^{n^{+}}$. For all $u, v$,

$$
\left(D_{u+v}+i\right)-\left(D_{u}+i\right)=D_{u+v}-D_{u},
$$

and to apply Kato's stability property, we need to give a relative bound on $D_{u+v}-D_{u}$, expressed in terms of $D_{u}+i$. We are going to obtain this using the relation (3.5). Indeed, since we know that $D$ is selfadjoint, $\langle D \xi, \xi\rangle=\langle\xi, D \xi\rangle$ and thus

$$
\|(D+i) \xi\|^{2}=\|D \xi\|^{2}+\|\xi\|^{2} .
$$

which shows that $\|D \xi\| \leqslant\|(D+i) \xi\|$. From this fact and (3.5), we deduce

$$
\left\|\left(D_{u+v}-D_{u}\right)(\underline{\omega})\right\| \leqslant o_{v}(1)\left\|\left(D_{u}+i\right) \underline{\omega}\right\|+o_{v}(1)\|\underline{\omega}\|,
$$

which let us apply $\left[39\right.$, Theorem 1.16, p. 196] to $D_{u}+i$ and $D_{u+v}-D_{u}$, leading to the expression

$$
\left(D_{u+v}+i\right)^{-1}=\left(D_{u}+i\right)^{-1}\left(1+\left(D_{u+v}-D_{u}\right)\left(D_{u}+i\right)^{-1}\right)^{-1},
$$

where both $\left(D_{u+v}-D_{u}\right)\left(D_{u}+i\right)^{-1}$ and $\left(1+\left(D_{u+v}-D_{u}\right)\left(D_{u}+i\right)^{-1}\right)^{-1}$ are bounded operators. This expression shows that $\left(D_{u+v}+i\right)^{-1}$ is a product of $\left(D_{u}+i\right)^{-1}$ in the ideal $\mathscr{L}^{n^{+}}$and a bounded operator. It is therefore itself in the ideal $\mathscr{L}^{n^{+}}$and this completes the proof.

The operator $d_{u}$ of Proposition 3.6 induces a cochain complex:

Proposition 3.9. The operator $d_{u}:=K_{u} d K_{-u}$, defined from $d=\Pi_{+} D$ on the domain of selfadjointness of $D_{u}$ is closable. Taking its closure, there is a cochain complex $\left(d_{u}, \mathscr{H}_{k}\right)$

$$
0 \rightarrow \mathscr{H}_{0} \stackrel{d_{u, 0}}{\longrightarrow} \mathscr{H}_{1} \rightarrow \cdots \rightarrow \mathscr{H}_{n-1} \stackrel{d_{u, n-1}}{\longrightarrow} \mathscr{H}_{n} \rightarrow 0
$$

Remark 3.10. In the complex (3.10), the map $d_{u, k}: \mathscr{H}_{k} \rightarrow \mathscr{H}_{k+1}$ is of course (the closure of) the restriction of $d_{u}$ to $\mathscr{H}_{k} \cap \operatorname{Dom}\left(D_{u}\right)$, where $\operatorname{Dom}\left(D_{u}\right)$ is the domain of selfadjointness of $D_{u}$. 
Proof. We first treat the case of $d$ (for $h=0$ ). In this case, if $x_{n} \rightarrow x$ and $y_{n} \rightarrow x$ while both $d x_{n}$ and $d y_{n}$ converge, we want to prove that $\lim d x_{n}=\lim d y_{n}$. Consider any $z \in \mathscr{H}$ which lives in a finite-dimensional vector space $V \otimes \wedge^{\bullet} \mathfrak{g}^{*}$ obtained from the Peter-Weyl decomposition. This ensures that $\Pi_{+} z$ is in $V \otimes \wedge^{\bullet} \mathfrak{g}^{*}$ and thus in the domain of $D$. We then have

$$
\left\langle z, \Pi_{+} D x_{n}\right\rangle=\left\langle D \Pi_{+} z, x_{n}\right\rangle \rightarrow\left\langle D \Pi_{+} z, x\right\rangle \leftarrow\left\langle z, \Pi_{+} D y_{n}\right\rangle
$$

Since we know that both $d x_{n}$ and $d y_{n}$ converge in $\mathscr{H}$ and that $\mathscr{D}$, the algebraic direct sum of all $V \otimes \bigwedge^{\bullet} \mathfrak{g}^{*}$ is dense, it is necessary that $\lim d x_{n}=\lim d y_{n}$ and this proves that $d$ is closable.

It follows that the kernel $\operatorname{ker}(\bar{d})$ is closed. Since $\mathcal{A}^{1} \otimes \bigwedge^{\bullet} \mathfrak{g}^{*}$ is a core for $D$, any $x$ in the domain $\operatorname{Dom}(\bar{d})$ can be approximated by $x_{n} \in \mathcal{A}^{1} \otimes \bigwedge^{\bullet} \mathfrak{g}^{*}$ such that $x_{n} \rightarrow x$ and $d x_{n} \rightarrow d x$. The density of $\mathcal{A}^{\infty}$ inside $\mathcal{A}^{1}$ (as discussed at the end of Section 2) then provides an approximation of the original $x \in \operatorname{dim}(\bar{d})$ by $y_{n} \in \mathcal{A}^{\infty} \otimes \Lambda^{\bullet} \mathfrak{g}^{*}=\Omega^{\bullet}$. For this sequence $y_{n}$, we know from Section 3 that $d^{2} y_{n}=0$. By density, we obtain that (3.10) is a cochain complex.

Similarly, for $d_{u}=K_{u} d K_{-u}$ if $x_{n} \rightarrow x$ and $y_{n} \rightarrow x$ while both $d_{u} x_{n}$ and $d_{u} y_{n}$ converge, we have $K_{-u} x_{n} \rightarrow K_{-u} x \leftarrow K_{-u} y_{n}$ and $K_{-u} d_{u} x_{n}=d K_{-u} x_{n}, K_{-u} d_{u} y_{n}=d K_{-u} y_{n}$. Since $d$ is closable, we get $\lim K_{-u} d_{u} x_{n}=\lim K_{-u} d_{u} y_{n}$, which suffices to prove that $d_{u}$ is also closable. The cochain property then follows from $d_{u}^{2}=K_{u} d^{2} K_{-u}=0$.

In the rest of this section, we will be interested in the reduced cohomology of the complex (3.10), namely the cohomology groups

$$
H^{k}\left(d_{u}, \mathscr{H}_{k}\right):=\operatorname{ker}\left(d_{u, k}\right) / \overline{\operatorname{Ran}\left(d_{u, k-1}\right)} .
$$

For any $u \in[0,1]$, let us write $E_{0, u}$ for the kernel of $D_{u}$. We have the following Hodge decomposition theorem for the conformally perturbed metric:

Theorem 3.11. Let $G$ be a compact Lie group of dimension $n$ acting ergodically on a unital $C^{*}$-algebra $A$. With the notations introduced previously, for any parameter $u \in[0,1]$, there is a decomposition of $\mathscr{H}$ into a direct sum of orthogonal Hilbert spaces

$$
\mathscr{H}=E_{-, u} \oplus E_{0, u} \oplus E_{+, u} .
$$

Proof. The operator $D_{u}$ is selfadjoint with compact resolvent, as a consequence of Proposition 3.6 and Corollary 3.8. Thus, we have an orthogonal sum $\mathscr{H}=E_{0, u} \oplus \overline{\operatorname{Ran}\left(D_{u}\right)}$. Following Proposition 3.6, $\overline{\operatorname{Ran}\left(D_{u}\right)}=E_{-, u} \oplus E_{+, u}$ and the sum is orthogonal, which proves the result.

We call the restriction of $D_{u}^{2}$ to $\mathscr{H}_{k}$ the Laplacian on $\mathscr{H}_{k}$ and denote it by $\Delta_{k}$, which is thus an unbounded operator on $\mathscr{H}_{k}$, defined on the domain $\mathcal{A}^{\infty} \otimes \bigwedge^{k} \mathfrak{g}^{*}$. Note that $\Delta_{k}$ actually depends on our choice of conformal perturbation $h \in \mathcal{A}^{1}$.

Corollary 3.12. Let $H^{k}\left(d_{u}, \mathscr{H}_{k}\right)$ be the cohomology groups introduced in (3.11), they identify naturally with the kernel of $\Delta_{k}$, i.e.,

$$
\operatorname{ker}\left(\Delta_{k}\right) \simeq H^{k}\left(d_{u}, \mathscr{H}_{k}\right)
$$

Remark 3.13. This Corollary implies in particular that these cohomology groups are finitedimensional, since $\operatorname{ker}\left(\Delta_{k}\right)=\operatorname{ker}\left(D_{u}\right)$ and $D_{u}$ has compact resolvent by Corollary 3.8.

Proof. The cohomology group $H^{k}\left(d_{u}, \mathscr{H}_{k}\right)$ is defined as $\operatorname{ker}\left(d_{u, k}\right) / \overline{\operatorname{Ran}\left(d_{u, k-1}\right)}$. The Hodge decomposition Theorem 3.11 can be combined with the projections $\Pi_{ \pm}$and $\Pi_{k}$ on $\mathscr{H}_{k}$ to prove that $\mathscr{H}_{k}=\operatorname{ker}\left(\Delta_{k}\right) \oplus \overline{\operatorname{Ran}\left(d_{u, k-1}\right)} \oplus \overline{\operatorname{Ran}\left(d_{u, k}^{*}\right)}$. We know that $\operatorname{ker}\left(d_{u, k}\right)=\operatorname{Ran}\left(d_{u, k}^{*}\right)^{\perp}$. Therefore $\operatorname{ker}\left(d_{u, k}\right)=\overline{\operatorname{Ran}\left(d_{u, k-1}\right)} \oplus \operatorname{ker}\left(\Delta_{k}\right)$ from which it follows immediately that $H^{k}\left(d_{u}, \mathscr{H}_{k}\right)=$ $\operatorname{ker}\left(d_{u, k}\right) / \overline{\operatorname{Ran}\left(d_{u, k-1}\right)} \simeq \operatorname{ker}\left(\Delta_{k}\right)$. 
Proposition 3.14. The cohomology groups $H^{k}\left(d_{u}, \mathscr{H}_{k}\right)$ are abstractly isomorphic to the nonperturbed $(h=0)$ cohomology groups $H^{k}\left(d, \mathscr{H}_{k}\right)$.

Proof. It is easy to check that $\operatorname{ker}\left(d_{u}\right)=K_{u} \operatorname{ker}(d)$ and $E_{+, u}=K_{u} E_{+, 0}$. Thus, as abstract vector space, $\operatorname{ker}\left(d_{u}\right) / E_{+, u}=K_{u} \operatorname{ker}\left(d_{0}\right) / K_{u} E_{+, 0}$ is finite-dimensional, with the same dimension as $\operatorname{ker}\left(d_{0}\right) / E_{+, 0}$.

Remark 3.15. The dimensions of $\operatorname{ker}\left(d_{u}\right) / E_{+, u}$ and $\operatorname{ker}\left(d_{0}\right) / E_{+, 0}$ are the same, but there are not "concretely isomorphic" for the scalar product we consider. The concrete realisation of $\operatorname{ker}\left(d_{u}\right) / E_{+, u}$ is $\left\{\underline{\omega} \in \operatorname{ker}\left(d_{u}\right): \forall \underline{\omega}^{\prime} \in E_{+, u},\left\langle\underline{\omega}, \underline{\omega}^{\prime}\right\rangle=0\right\}$. However, $K_{u}$ does not preserve scalar products and therefore, $\operatorname{ker}\left(d_{u}\right) / E_{+, u}$ is not realised concretely by $K_{u} E_{0,0}$. In other words, $K_{u} E_{0,0}$ is not the space of harmonic forms for $D_{u}$.

\section{Conformally twisted spectral triples for $C^{*}$-dynamical systems}

In the following theorem, we use the selfadjoint operator $D_{u}$ to construct spectral triples for the natural actions of the algebra $A$ (with its left action on $\mathscr{H}$ ) and the algebra $A^{\text {op }}$ (acting on the right of $\mathscr{H})$.

Theorem 4.1. Let $G$ be a compact Lie group of dimension $n$ acting ergodically on a unital $C^{*}$-algebra $A$, then using the unique $G$-invariant trace $\varphi_{0}$ of Theorem 2.2 , we write $\mathscr{H}_{0}:=$ $\operatorname{GNS}\left(A, \varphi_{0}\right)$.

For any fixed $h \in \mathcal{A}^{1}$ and any $u \in[0,1]$, the data $\left(A, \mathscr{H}_{0} \otimes \wedge^{\bullet} \mathfrak{g}^{*}, D_{u}\right)$ with grading $\gamma$ defines an even $n^{+}$-summable spectral triple, where

- the representation $\pi$ of $A$ on $\mathscr{H}=\mathscr{H}_{0} \otimes \wedge^{\bullet} \mathfrak{g}^{*}$ is given by restriction of the left multiplication (3.2);

- the unbounded operator $D_{u}$ is the unique selfadjoint extension of

$$
D_{u}=K_{u} d K_{-u}+K_{-u} d^{*} K_{u},
$$

defined on the core $C=\mathcal{A}^{1} \otimes \bigwedge^{\bullet} \mathfrak{g}^{*}$, the operator $K_{u}$ being defined by (3.4);

- the grading operator $\gamma$ is defined on degree $k$ forms by

$$
\gamma\left(a \otimes v_{1} \wedge \cdots \wedge v_{k}\right)=(-1)^{k}\left(a \otimes v_{1} \wedge \cdots \wedge v_{k}\right) .
$$

For any fixed $h \in \mathcal{A}^{1}$ and any $u \in[0,1]$, the data $\left(A^{o p}, \mathscr{H}_{0} \otimes \bigwedge^{\bullet} \mathfrak{g}^{*}, D_{u}\right)$ with grading $\gamma$ defines an even $n^{+}$-summable twisted spectral triple, with the automorphism $\beta$ on A given by $\beta(a)=$ $e^{h u} a e^{-h u}-$ we use this $\beta$ to define an automorphism on $A^{o p}$.

Remark 4.2. The morphism $\beta$ defined above preserves the multiplication of $A^{\text {op }}$. It also satisfies the relation unitarity condition (see [16, equation $(3.4)]$ ) that is $\beta\left(\left(a^{\mathrm{op}}\right)^{*}\right)=\left(\beta^{-1}\left(a^{\mathrm{op}}\right)\right)^{*}$.

Proof. It is clear from the definition of $\pi$ that $A$ is represented on $\mathscr{H}$ by bounded operators. The existence and uniqueness of the selfadjoint extension of $D_{u}$ is proved in Proposition 3.6, while the compact resolvent and finite summability properties are shown in Corollary 3.8.

We now prove that the commutator of $D_{u}$ with $a \in \mathcal{A}^{1}$ is bounded. To this end, we use the notations of Lemma 3.2 to decompose the operator $D_{u}$. We call

- Part (0) is the "bounded part" of $D_{u}$, that is the terms

$$
-K_{u}\left(\frac{1}{2} \sum_{k, \alpha, \beta} c_{\alpha \beta}^{i_{k}} \otimes B_{\alpha, \beta}^{i_{k}}\right) K_{-u} \quad \text { and } \quad-K_{-u}\left(\frac{1}{2} \sum_{k, \alpha, \beta} \overline{c_{\alpha, \beta}^{i_{k}}} \otimes\left(B_{\alpha, \beta}^{i_{k}}\right)^{*}\right) K_{u} .
$$


- Part (I) consists of the terms

$$
K_{u}\left(\sum_{j} \partial_{j} \otimes T_{j}\right) K_{-u} .
$$

- Part (II) consists of the terms

$$
K_{-u}\left(\sum_{j} \partial_{j} \otimes T_{j}^{*}\right) K_{u} .
$$

Part (0) commutes with the left multiplication by $a \in \mathcal{A}^{1}$, and thus it does not contribute to the commutator. We therefore only need to estimate Parts (I) and (II) of $D_{u}\left(a^{\prime} \underline{\omega}\right)$ for $\underline{\omega}=a \otimes v_{1} \wedge \cdots \wedge v_{k}$, that is

$$
\begin{aligned}
\sum_{j} \partial_{j}\left(a^{\prime} a e^{-(n / 2-k) h u}\right) e^{(n / 2-(k+1)) h u} \otimes T_{j}\left(v_{1} \wedge \cdots \wedge v_{k}\right) \\
\quad+\sum_{j} \partial_{j}\left(a^{\prime} a e^{(n / 2-k) h u}\right) e^{-(n / 2-(k-1)) h u} \otimes T_{j}^{*}\left(v_{1} \wedge \cdots \wedge v_{k}\right) \\
=\sum_{j}\left(\partial_{j}\left(a^{\prime}\right) a e^{-(n / 2-k) h u}+a^{\prime} \partial_{j}\left(a e^{-(n / 2-k) h u}\right)\right) e^{(n / 2-(k+1)) h u} \otimes T_{j}\left(v_{1} \wedge \cdots \wedge v_{k}\right) \\
\quad+\sum_{j}\left(\partial_{j}\left(a^{\prime}\right) a e^{(n / 2-k) h u}+a^{\prime} \partial_{j}\left(a e^{(n / 2-k) h u}\right)\right) e^{-(n / 2-(k-1)) h u} \otimes T_{j}^{*}\left(v_{1} \wedge \cdots \wedge v_{k}\right) .
\end{aligned}
$$

It follows from these considerations that

$$
\left[D_{u}, a^{\prime}\right] \underline{\omega}=\sum_{j} \partial_{j}\left(a^{\prime}\right) a e^{-h u} \otimes\left(T_{j}+T_{j}^{*}\right)\left(v_{1} \wedge \cdots \wedge v_{k}\right),
$$

which is clearly a bounded function of $\underline{\omega}$ for any $a^{\prime} \in \mathcal{A}^{1}$. Moreover, such $a^{\prime} \in \mathcal{A}^{1}$ sends the core $C$ of our selfadjoint operator $D_{u}$ to itself and following [48, Proposition A.1, p. 293], this suffices to ensure that $a^{\prime} \in \mathcal{A}^{1}$ sends the domain of $D_{u}$ to itself. The algebra $\mathcal{A}$ of Definition 2.4 thus contains $\mathcal{A}^{1}$ and is dense in the $C^{*}$-algebra $A$. This completes the proof that $\left(A, \mathscr{H}, D_{u}\right)$ is a $n^{+}$-summable spectral triple.

It remains to study its parity: it is clear from the definition that $\gamma$ sends the core $C$ to itself and thus it leaves the full domain of the selfadjoint operator $D_{u}$ stable. Clearly, $\gamma$ distinguishes only between $\mathscr{H}_{\text {even }}:=A \otimes \bigwedge^{\text {even }} \mathfrak{g}^{*}$ and $\mathscr{H}_{\text {odd }}:=A \otimes \bigwedge^{\text {odd }} \mathfrak{g}^{*}$ and $\pi(a)$ leaves both spaces invariant, while $D_{u}$ is an odd operator. This proves that $\left(A, \mathscr{H}, D_{u}\right)$ with $\gamma$ is an even spectral triple.

The parity paragraph above applies verbatim to the spectral triple constructed from the right action of $A^{\mathrm{op}}$. The summability property is also conserved. It remains to investigate the bounded twisted commutators. Notice first that if $a^{\prime}, h \in \mathcal{A}^{1}$ then both right multiplications by $a^{\prime}$ and by $\beta\left(a^{\prime}\right)$ leave the core $C$ of $D_{u}$ invariant and therefore the domain of $D_{u}$ is also stable under these right multiplication.

Using the decomposition of $D_{u}$ into Parts (0), (I) and (II), it appears that Part (0) commutes with the right action of $A^{\text {op }}$ and therefore does not contribute to the commutator. We treat Parts (I) and (II) separately. Keeping only Part (I) in the expression $D_{u}\left(\underline{\omega} \cdot a^{\prime}\right)$ for $\underline{\omega}=a \otimes v_{1} \wedge \cdots \wedge v_{k}$, we get

$$
\begin{aligned}
\sum_{j} \partial_{j}\left(a a^{\prime} e^{-(n / 2-k) h u}\right) e^{(n / 2-(k+1)) h u} \otimes T_{j}\left(v_{1} \wedge \cdots \wedge v_{k}\right) \\
\quad=\sum_{j}\left(\partial_{j}(a) a^{\prime} e^{-(n / 2-k) h u}+a \partial_{j}\left(a^{\prime}\right) e^{-(n / 2-k) h u}\right) e^{(n / 2-(k+1)) h u} \otimes T_{j}\left(v_{1} \wedge \ldots \wedge v_{k}\right)
\end{aligned}
$$




$$
\begin{aligned}
& +\sum_{j} a a^{\prime} \partial_{j}\left(e^{-(n / 2-k) h u}\right) e^{(n / 2-(k+1)) h u} \otimes T_{j}\left(v_{1} \wedge \cdots \wedge v_{k}\right) \\
= & \sum_{j}\left(\partial_{j}(a) a^{\prime} e^{-h u}+a \partial_{j}\left(a^{\prime}\right) e^{-h u}+a a^{\prime} \partial_{j}\left(e^{-(n / 2-k) h u}\right) e^{(n / 2-(k+1)) h u}\right) \\
& \otimes T_{j}\left(v_{1} \wedge \cdots \wedge v_{k}\right) .
\end{aligned}
$$

We compare this expression to $D_{u}(\underline{\omega}) \beta\left(a^{\prime}\right)$, i.e.,

$$
\begin{aligned}
\sum_{j} \partial_{j}\left(a e^{-(n / 2-k) h u}\right) e^{(n / 2-(k+1)) h u} e^{h u} a^{\prime} e^{-h u} \otimes T_{j}\left(v_{1} \wedge \cdots \wedge v_{k}\right) \\
=\sum_{j} \partial_{j}(a) a^{\prime} e^{-h u} \otimes T_{j}\left(v_{1} \wedge \cdots \wedge v_{k}\right) \\
\quad+\sum_{j} a \partial_{j}\left(e^{-(n / 2-k) h u}\right) e^{(n / 2-(k+1)) h u} e^{h u} a^{\prime} e^{-h u} \otimes T_{j}\left(v_{1} \wedge \cdots \wedge v_{k}\right) .
\end{aligned}
$$

In these two sums, the only terms that could lead to an unbounded contribution are those containing $\partial_{j}(a)$, but these two terms cancel. At this point, we must perform the same computation on Part (II) to make sure that the automorphism $\beta$ is also suitable for this case. A very similar computation proves that this it is indeed the case - the key property is that $e^{-(n / 2-k) h u} e^{(n / 2-(k+1)) h u}=e^{-h u}=e^{(n / 2-k) h u} e^{-(n / 2-(k-1)) h u}-$ and thus the operator (defined a priori only on $C$ )

$$
D_{u} \pi^{\mathrm{op}}\left(\left(a^{\prime}\right)^{\mathrm{op}}\right)-\pi^{\mathrm{op}}\left(\beta\left(a^{\prime}\right)^{\mathrm{op}}\right) D_{u},
$$

where $\pi^{\mathrm{op}}\left(\left(a^{\prime}\right)^{\mathrm{op}}\right)=\mathcal{R}_{a^{\prime}} \otimes \operatorname{Id}_{\wedge^{\bullet} \mathfrak{g}^{*}}$, extends to a bounded operator on $\mathscr{H}$.

Since the operator $D_{u}$ is odd with respect to the grading operator $\gamma$, we can write $D_{u}$ as combination of $D_{u}^{+}: \mathscr{H}_{\text {even }} \rightarrow \mathscr{H}_{\text {odd }}$ and $D_{u}^{-}: \mathscr{H}_{\text {odd }} \rightarrow \mathscr{H}_{\text {even }}$. The odd Fredholm operator admits a (possibly) nontrivial index defined as

$$
\operatorname{Index}_{\text {odd }}\left(D_{u}\right)=\operatorname{dim} \operatorname{ker}\left(D_{u}^{+}\right)-\operatorname{dim} \operatorname{ker}\left(D_{u}^{-}\right)
$$

(see, e.g., [32, equation (9.36), p. 397]).

\section{Existence of a Chern-Gauss-Bonnet theorem for conformal perturbations of $C^{*}$-dynamical systems}

In this section we show that the Hodge decomposition theorem proved in Section 3 indicates the existence of an analog of the Chern-Gauss-Bonnet theorem for the $C^{*}$-dynamical systems studied in the present article. Let us explain the classical case before stating the statement for our setting. Indeed, because of the natural isomorphism between the space of harmonic differential forms and the de Rham cohomology groups, for a classical closed manifold $M$, the index of the operator $d+d^{*}: \Omega^{\text {even }} M \rightarrow \Omega^{\text {odd }} M$ is equal to the Euler characteristic of $M$. On the other hand the McKean-Singer index theorem asserts that the index is given by

$$
\operatorname{Index}\left(d+d^{*}: \Omega^{\text {even }} \rightarrow \Omega^{\text {odd }}\right)=\sum_{i=0}^{\operatorname{dim} M}(-1)^{i} \operatorname{Tr}\left(e^{-t \triangle_{i}}\right),
$$

where $\triangle_{i}=d^{*} d+d d^{*}$ is the Laplacian on the space of $i$-differential forms on $M$, and $t$ is any positive number. This formula, furthermore, contains local geometric information as $t \rightarrow 0^{+}$, 
since there is a small time asymptotic expansion of the form

$$
\operatorname{Tr}\left(e^{-t \triangle_{i}}\right) \sim t^{-\operatorname{dim} M / 2} \sum_{j=0}^{\infty} a_{2 j}\left(\triangle_{i}\right) t^{j}
$$

The coefficients $a_{2 j}\left(\triangle_{i}\right)$ are local geometric invariants, which depend on the high frequency behaviour of the eigenvalues of the Laplacian and are the integrals of some invariantly defined local functions $a_{2 j}\left(x, \triangle_{i}\right)$ against the volume form of $M$. Independence of the index from $t$ implies that the alternating sum of the constant terms in the above asymptotic expansions for $\triangle_{i}$ gives the index. Hence, using the Hodge decomposition theorem,

$$
\chi(M)=\operatorname{Index}\left(d+d^{*}: \Omega^{\mathrm{even}} \rightarrow \Omega^{\mathrm{odd}}\right)=\int_{M} \sum_{i=0}^{\operatorname{dim} M}(-1)^{i} a_{\operatorname{dim} M}\left(x, \triangle_{i}\right) d \operatorname{vol}_{g} .
$$

In fact, the integrand in the latter coincides with the Pfaffian of the curvature form, which is a remarkable and difficult identification [2].

With notations and assumptions as in Section 3, we obtain the following result which indicates the existence of an analog of the Chern-Gauss-Bonnet theorem in the setting of $C^{*}$-dynamical systems studied in this article.

Theorem 5.1. The Euler characteristic $\chi$ of the complex $\left(d_{u}, \mathscr{H}_{k}\right)$ is related to the odd index defined in (4.1)

$$
\chi=\sum_{k=0}^{n}(-1)^{k} \operatorname{dim} H^{k}\left(d_{u}, \mathscr{H}_{k}\right)=\sum_{k=0}^{n}(-1)^{k} \operatorname{ker}\left(\Delta_{k}\right)=\operatorname{Index}_{\text {odd }}\left(D_{u}\right),
$$

and is independent of the conformal factor $e^{-h}$.

Proof. The first equality is actually the definition of the Euler characteristic $\chi$. The second equality is an immediate consequence of Corollary 3.12. The third equality and the last statement can be justified by using Remark 3.13 and Proposition 3.14. That is, $\underline{\omega} \in \operatorname{ker}\left(\Delta_{k}\right)$ means in particular that $\underline{\omega}$ is in the domain of $\Delta_{k}$, which is included in the domain of $D_{u}$ (by definition). We then have

$$
0=\left\langle\underline{\omega}, \Delta_{k} \underline{\omega}\right\rangle=\left\langle D_{u} \underline{\omega}, D_{u} \underline{\omega}\right\rangle,
$$

which proves that $\underline{\omega} \in \operatorname{ker}\left(D_{u}\right)$. For a $k$-form $\underline{\omega}$, the converse is obvious. It follows that $\operatorname{ker}\left(D_{u}^{+}\right)=\bigoplus_{k \geqslant 0} \operatorname{ker}\left(\Delta_{2 k}\right)$ and $\operatorname{ker}\left(D_{u}^{-}\right)=\bigoplus_{k \geqslant 0} \operatorname{ker}\left(\Delta_{2 k+1}\right)$, which yields

$$
\operatorname{Index} \operatorname{odd}_{(}\left(D_{u}\right)=\operatorname{dim} \operatorname{ker}\left(D_{u}^{+}\right)-\operatorname{dim} \operatorname{ker}\left(D_{u}^{-}\right)=\bigoplus_{k \geqslant 0} \operatorname{dim} \operatorname{ker}\left(\Delta_{2 k}\right)-\bigoplus_{k \geqslant 0} \operatorname{ker}\left(\Delta_{2 k+1}\right)=\chi
$$

The dimension of these groups are independent of the conformal factor $e^{-h}$ as a consequence of Proposition 3.14.

Remark 5.2. An alternative proof of the index property using only bounded operators can be obtained using Sobolev spaces. For a clear account of these spaces and their analytic properties in our setting, we refer the reader to the paper [55]. Also, in order to have a complete analog of the Chern-Gauss-Bonnet theorem, one needs to find a local geometric formula for the index, which is proved above to be a conformal invariant. 
The heat kernels of Laplacians of conformally perturbed metrics on certain noncommutative spaces such as the noncommutative $n$-tori $\mathbb{T}_{\Theta}^{n}$ admit asymptotic expansions of the form

$$
\operatorname{Tr}\left(e^{-t \Delta_{k}}\right) \sim \sum_{j=0}^{\infty} a_{j}\left(\Delta_{k}\right) t^{(j-n) / 2}, \quad t \rightarrow 0^{+} .
$$

In fact, for noncommutative tori, each Laplacian $\Delta_{k}$ is an elliptic selfadjoint differential operator of order 2, and asymptotic expansions of this form can be derived by using the heat kernel method explained in [31] while employing Connes' pseudodifferential calculus [12]. This method was indeed used in $[17,18,25,26,28]$, for calculating and studying the term in the expansion that is related to the scalar curvature of noncommutative two and four tori. Going through this process for noncommutative tori $\mathbb{T}_{\Theta}^{n}$, one can see that the odd coefficients in the latter asymptotic expansion will vanish, since in their explicit formula in terms of the pseudodifferential symbol of $\Delta_{k}$, there is an integration over the Euclidean space $\mathbb{R}^{n}$ of an odd function involved (see [31, p. 54 and Theorem 1.7.6, p. 58]). Thus in the case of the noncommutative torus $\mathbb{T}_{\Theta}^{n}$ we can write (5.1) as

$$
\operatorname{Tr}\left(e^{-t \Delta_{k}}\right) \sim t^{-n / 2} \sum_{j=0}^{\infty} a_{2 j}\left(\Delta_{k}\right) t^{j}, \quad t \rightarrow 0^{+} .
$$

Now, using the McKean-Singer index formula [31, Lemma 1.6.5, p. 47] and our analog of Hodge decomposition theorem, for any $t>0$ we have

$$
\chi=\sum_{k=0}^{n}(-1)^{k} \operatorname{Tr}\left(e^{-t \Delta_{k}}\right) .
$$

Thus from equations (5.2) and (5.3), and using the independence of the Euler characteristic from $t$ which implies that only the constant term from (5.2) contributes to the calculation of the Euler characteristic, we can write

$$
\chi=\sum_{k=0}^{n}(-1)^{k} a_{n}\left(\Delta_{k}\right)=\sum_{k=0}^{n}(-1)^{k} \varphi_{0}\left(\mathcal{R}_{k}\right),
$$

where the local geometric invariants $\mathcal{R}_{k}$ are derived from the pseudodifferential symbols of the Laplacian $\Delta_{k}$, by a heat kernel method. This method was used for example in $[17,26,28]$ for

computation of scalar curvature for noncommutative two and four tori. The alternating sum of the $\mathcal{R}_{k}$ gives a noncommutative analog of the local expression for the Euler class.

\section{Summary and conclusions}

The Chern-Gauss-Bonnet theorem is an important generalization of the Gauss-Bonnet theorem for surfaces, which states that the Euler characteristic of an even-dimensional Riemannian manifold can be computed as the integral of a characteristic class, namely the Pfaffian of the curvature form, which is a local invariant of the geometry. In particular, it shows that the integral of this geometric invariant is independent of the metric and depends only on the topology of the manifold. The results obtained in this paper show that the analog of this theorem holds for a general ergodic $C^{*}$-dynamical system, whose algebra and Lie group are not necessarily commutative. To be more precise, the family of metrics considered for a dynamical system is obtained by using an invertible positive element of the $C^{*}$-algebra to conformally perturb a fixed metric defined via the unique invariant trace, and our result is about the invariance of a quantity, which is a natural analog of the Euler characteristic, from the conformal factor. 
This type of results were previously proved for the noncommutative two torus $\mathbb{T}_{\theta}^{2}$. That is, the analog of the Gauss-Bonnet theorem was proved in [18] and extended to general translation invariant complex structures on these very important but particular $C^{*}$-algebras in [25], where a conformal factor varies the metric. The differential geometry of $C^{*}$-dynamical systems were developed and studied in [12], where the noncommutative two torus $\mathbb{T}_{\theta}^{2}$ played a crucial role. However the investigation of the analog of the Gauss-Bonnet theorem for $\mathbb{T}_{\theta}^{2}$, when the flat metric is conformally perturbed was pioneered in [11], where after heavy calculations, some noncommutative features seemingly indicated that the theorem does not hold. However, studying the spectral action in the presence of a dilaton [10], the development of the theory of twisted spectral triples [16], and further studies of examples of complex structures on noncommutative manifolds [40], led to convincing observations that the Gauss-Bonnet theorem holds for the noncommutative two torus. Then, by further analysis of the expressions and functions of a modular automorphism obtained in [11], Connes and Tretkoff proved the desired result in [18] for the simplest translation invariant conformal structure, and the generalization of their result was established in [25] (where the use of a computer for the heavy computations was inevitable).

It is remarkable that, a non-computational proof of the Gauss-Bonnet theorem for the noncommutative two torus is given in [17], which is based on the work [4], where the conformal index of a Riemannian manifold is defined using properties of conformally covariant operators and the variational properties of their spectral zeta functions. Therefore, since computations are enormously more involved in dimensions higher than two, it is of great importance to use spectral methods to show the existence of the analog of the Chern-Gauss-Bonnet theorem, which is presented in this article, not only for nocommutative tori, but for general $C^{*}$-dynamical systems. We have also paid special attention to the spectral properties of the analog of the Hodgede Rham operator of the perturbed metric: we have proved its selfadjointness and shown that the spectral dimension is preserved. We have then shown that this operator gives rise to a spectral triple with the unitary left action of the algebra, and gives a twisted spectral triple with the unitary action of the opposite algebra on the right, generalizing the construction in [18] on the noncommutative two torus and providing abstractly a large family of twisted spectral triples.

\section{Acknowledgements}

The authors thank the Hausdorff Research Institute for Mathematics (HIM) for their hospitality and support during the trimester program on Noncommutative Geometry and its Applications in 2014, where the present work was partially carried out. They also thank the anonymous referees for their constructive feedback. Parts of this article were obtained and written while the second author was working as a postdoc at the University of Glasgow. He would like to thank C. Voigt for enabling his stay in Scotland.

\section{References}

[1] Albeverio S., Høegh-Krohn R., Ergodic actions by compact groups on $C^{*}$-algebras, Math. Z. 174 (1980), $1-17$.

[2] Atiyah M., Bott R., Patodi V.K., On the heat equation and the index theorem, Invent. Math. 19 (1973), 279-330.

[3] Bhuyain T.A., Marcolli M., The Ricci flow on noncommutative two-tori, Lett. Math. Phys. 101 (2012), 173-194, arXiv:1107.4788.

[4] Branson T.P., Ørsted B., Conformal indices of Riemannian manifolds, Compositio Math. 60 (1986), $261-293$.

[5] Bratteli O., Derivations, dissipations and group actions on $C^{*}$-algebras, Lecture Notes in Math., Vol. 1229, Springer-Verlag, Berlin, 1986.

[6] Carey A.L., Neshveyev S., Nest R., Rennie A., Twisted cyclic theory, equivariant $K K$-theory and KMS states, J. Reine Angew. Math. 650 (2011), 161-191, arXiv:0808.3029. 
[7] Carey A.L., Phillips J., Rennie A., Semifinite spectral triples associated with graph $C^{*}$-algebras, in Traces in Number Theory, Geometry and Quantum Fields, Aspects Math., Vol. E38, Friedr. Vieweg, Wiesbaden, 2008, 35-56, arXiv:0707.3853.

[8] Carey A.L., Phillips J., Rennie A., Twisted cyclic theory and an index theory for the gauge invariant KMS state on the Cuntz algebra $O_{n}, J$. K-Theory 6 (2010), 339-380, arXiv:0801.4605.

[9] Chamseddine A.H., Connes A., The spectral action principle, Comm. Math. Phys. 186 (1997), 731-750, hep-th/9606001.

[10] Chamseddine A.H., Connes A., Scale invariance in the spectral action, J. Math. Phys. 47 (2006), 063504, 19 pages, hep-th/0512169.

[11] Cohen P.B., Connes A., Conformal geometry of the irrational rotation algebra, Preprint MPI, 1992.

[12] Connes A., $C^{*}$ algèbres et géométrie différentielle, C. R. Acad. Sci. Paris Sér. A-B 290 (1980), A599-A604, hep-th/0101093.

[13] Connes A., Noncommutative differential geometry, Inst. Hautes Études Sci. Publ. Math. 62 (1985), 257-360.

[14] Connes A., Noncommutative geometry, Academic Press, Inc., San Diego, CA, 1994.

[15] Connes A., Moscovici H., The local index formula in noncommutative geometry, Geom. Funct. Anal. 5 (1995), 174-243.

[16] Connes A., Moscovici H., Type III and spectral triples, in Traces in number theory, geometry and quantum fields, Aspects Math., Vol. E38, Friedr. Vieweg, Wiesbaden, 2008, 57-71, math.OA/0609703.

[17] Connes A., Moscovici H., Modular curvature for noncommutative two-tori, J. Amer. Math. Soc. 27 (2014), 639-684, arXiv:1110.3500.

[18] Connes A., Tretkoff P., The Gauss-Bonnet theorem for the noncommutative two torus, in Noncommutative Geometry, Arithmetic, and Related Topics, Johns Hopkins Univ. Press, Baltimore, MD, 2011, 141-158, arXiv:0910.0188.

[19] Cuntz J., Simple $C^{*}$-algebras generated by isometries, Comm. Math. Phys. 57 (1977), 173-185.

[20] Dąbrowski L., Sitarz A., An asymmetric noncommutative torus, SIGMA 11 (2015), 075, 11 pages, arXiv:1406.4645.

[21] Devastato A., Martinetti P., Twisted spectral triple for the standard model and spontaneous breaking of the grand symmetry, arXiv:1411.1320.

[22] Fathizadeh F., On the scalar curvature for the noncommutative four torus, J. Math. Phys. 56 (2015), 062303, 14 pages, arXiv:1410.8705.

[23] Fathizadeh F., Khalkhali M., The algebra of formal twisted pseudodifferential symbols and a noncommutative residue, Lett. Math. Phys. 94 (2010), 41-61, arXiv:0810.0484.

[24] Fathizadeh F., Khalkhali M., Twisted spectral triples and Connes' character formula, in Perspectives on Noncommutative Geometry, Fields Inst. Commun., Vol. 61, Amer. Math. Soc., Providence, RI, 2011, 79-101, arXiv:1106.6127.

[25] Fathizadeh F., Khalkhali M., The Gauss-Bonnet theorem for noncommutative two tori with a general conformal structure, J. Noncommut. Geom. 6 (2012), 457-480, arXiv:1005.4947.

[26] Fathizadeh F., Khalkhali M., Scalar curvature for the noncommutative two torus, J. Noncommut. Geom. 7 (2013), 1145-1183, arXiv:1110.3511.

[27] Fathizadeh F., Khalkhali M., Weyl's law and Connes' trace theorem for noncommutative two tori, Lett. Math. Phys. 103 (2013), 1-18, arXiv:1111.1358.

[28] Fathizadeh F., Khalkhali M., Scalar curvature for noncommutative four-tori, J. Noncommut. Geom. 9 (2015), 473-503, arXiv:1301.6135.

[29] Fathizadeh F., Wong M.W., Noncommutative residues for pseudo-differential operators on the noncommutative two-torus, J. Pseudo-Differ. Oper. Appl. 2 (2011), 289-302.

[30] Gabriel O., Grensing M., Ergodic actions and spectral triples, arXiv:1302.0426.

[31] Gilkey P.B., Invariance theory, the heat equation, and the Atiyah-Singer index theorem, Mathematics Lecture Series, Vol. 11, Publish or Perish, Inc., Wilmington, DE, 1984.

[32] Gracia-Bondía J.M., Várilly J.C., Figueroa H., Elements of noncommutative geometry, Birkhäuser Advanced Texts: Basler Lehrbücher, Birkhäuser Boston, Inc., Boston, MA, 2001.

[33] Greenfield M., Marcolli M., Teh K., Twisted spectral triples and quantum statistical mechanical systems, p-Adic Numbers Ultrametric Anal. Appl. 6 (2014), 81-104. 
[34] Higson N., The residue index theorem of Connes and Moscovici, in Surveys in noncommutative geometry, Clay Math. Proc., Vol. 6, Amer. Math. Soc., Providence, RI, 2006, 71-126.

[35] Høegh-Krohn R., Landstad M.B., Størmer E., Compact ergodic groups of automorphisms, Ann. of Math. 114 (1981), 75-86.

[36] Iochum B., Masson T., Crossed product extensions of spectral triples, J. Noncommut. Geom., to appear, arXiv:1406.4642.

[37] Kaad J., On modular semifinite index theory, arXiv:1111.6546.

[38] Kaad J., Senior R., A twisted spectral triple for quantum SU(2), J. Geom. Phys. 62 (2012), 731-739, arXiv:1109.2326.

[39] Kato T., Perturbation theory for linear operators, Classics in Mathematics, Springer-Verlag, Berlin, 1995.

[40] Khalkhali M., Landi G., van Suijlekom W.D., Holomorphic structures on the quantum projective line, Int. Math. Res. Not. 2011 (2011), 851-884, arXiv:0907.0154.

[41] Knapp A.W., Lie groups, Lie algebras, and cohomology, Mathematical Notes, Vol. 34, Princeton University Press, Princeton, NJ, 1988.

[42] Krýsl S., Hodge theory for elliptic complexes over unital $C^{*}$-algebras, Ann. Global Anal. Geom. 45 (2014), 197-210, arXiv:1309.4560.

[43] Krýsl S., Hodge theory for complexes over $C^{*}$-algebras with an application to A-ellipticity, Ann. Global Anal. Geom. 47 (2015), 359-372, arXiv:1309.4560.

[44] Krýsl S., Elliptic complexes over $C^{*}$-algebras of compact operators, J. Geom. Phys. 101 (2016), 27-37, arXiv:1506.06244.

[45] Lesch M., Moscovici H., Modular curvature and Morita equivalence, arXiv:1505.00964.

[46] Lord S., Sukochev F., Zanin D., Singular traces. Theory and applications, De Gruyter Studies in Mathematics, Vol. 46, De Gruyter, Berlin, 2013.

[47] Moscovici H., Local index formula and twisted spectral triples, in Quanta of Maths, Clay Math. Proc., Vol. 11, Amer. Math. Soc., Providence, RI, 2010, 465-500, arXiv:0902.0835.

[48] Paterson A.L.T., Contractive spectral triples for crossed products, Math. Scand. 114 (2014), 275-298, arXiv:1204.4404.

[49] Ponge R., Wang H., Noncommutative geometry and conformal geometry. III. Vafa-Witten inequality and Poincaré duality, Adv. Math. 272 (2015), 761-819, arXiv:1310.6138.

[50] Reed M., Simon B., Methods of modern mathematical physics. I. Functional analysis, Academic Press, Inc., New York, 1980.

[51] Reed M., Simon B., Methods of modern mathematical physics. II. Fourier analysis, selfadjointness, Academic Press, Inc., New York, 1975.

[52] Rieffel M.A., Metrics on states from actions of compact groups, Doc. Math. 3 (1998), 215-229, math.OA/9807084.

[53] Simon B., Trace ideals and their applications, Mathematical Surveys and Monographs, Vol. 120, 2nd ed., Amer. Math. Soc., Providence, RI, 2005.

[54] Størmer E., Spectra of ergodic transformations, J. Funct. Anal. 15 (1974), 202-215.

[55] Wahl C., Index theory for actions of compact Lie groups on $C^{*}$-algebras, J. Operator Theory 63 (2010), 217-242, arXiv:0707.3207.

[56] Wassermann A., Ergodic actions of compact groups on operator algebras. I. General theory, Ann. of Math. 130 (1989), 273-319.

[57] Wassermann A., Ergodic actions of compact groups on operator algebras. II. Classification of full multiplicity ergodic actions, Canad. J. Math. 40 (1988), 1482-1527.

[58] Wassermann A., Ergodic actions of compact groups on operator algebras. III. Classification for SU(2), Invent. Math. 93 (1988), 309-354.

[59] Wodzicki M., Local invariants of spectral asymmetry, Invent. Math. 75 (1984), 143-177.

[60] Wodzicki M., Noncommutative residue. I. Fundamentals, in $K$-Theory, Arithmetic and Geometry (Moscow, 1984-1986), Lecture Notes in Math., Vol. 1289, Springer, Berlin, 1987, 320-399. 